\title{
Tianwen-1 MINPA observations in the solar wind
}

\author{
AiBing Zhang 1,2,3,4*, LingGao Kong1,2,3,4*, WenYa Li1,5, Lei Li1,5, BinBin Tang1,5, ZhaoJin Rong 4,6, Yong Wei4,6, \\ JiJie Ma1,2,3,4, YiTeng Zhang 1,5, LiangHai Xie 1,5, YuXian Wang 1,5, JianSen He, Bin Liu' 1,2,3,4, WenJing Wang 1,2,3,4, \\ Bin Su1,2,3,4, JiaWei Li ${ }^{8}$, Xu Tan', Fang Wang9 ${ }^{9}$, TaiFeng Jin 1,4,5, FuHao Qiao ${ }^{1,4,5}$, Peter Wurz' ${ }^{10}$, Yan Zhu', \\ YunFei Bai ${ }^{1}$, YiRen Li ${ }^{11}$, XinBo Zhu ${ }^{12}$, YueQiang Sun ${ }^{1,2,3,4}$, YongLiao Zou' ${ }^{1}$, and Chi Wang 1,4,5 \\ ${ }^{1}$ National Space Science Center, Chinese Academy of Sciences, Beijing 100190, China; \\ ${ }^{2}$ Beijing Key Laboratory of Space Environment Exploration, Beijing 100190, China; \\ ${ }^{3}$ Key Laboratory of Science and Technology on Space Environment Situational Awareness, Chinese Academy of Sciences, Beijing 100190, China; \\ ${ }^{4}$ University of Chinese Academy of Sciences, Beijing 100049, China; \\ ${ }^{5}$ State Key Laboratory of Space Weather, Chinese Academy of Sciences, Beijing 100190, China; \\ ${ }^{6}$ Key Laboratory of Earth and Planetary Physics, Institute of Geology and Geophysics, Chinese Academy of Sciences, Beijing 100029, China; \\ ${ }^{7}$ Institute of Space Physics and Applied Technology, Peking University, Beijing 100871, China; \\ ${ }^{8}$ Lunar Exploration and Space Engineering Center, Beijing 100190, China; \\ ${ }^{9}$ National Astronomical Observatories, Chinese Academy of Sciences, Beijing 100012, China; \\ 10Physics Institute, University of Bern, Bern 3012, Switzerland; \\ ${ }^{11}$ University of Science and Technology of China, Hefei 230026, China; \\ ${ }^{12}$ Shanghai Academy of Spaceflight Technology, Shanghai 201109, China \\ Key Points: \\ - We present the first results of Tianwen-1/MINPA solar wind observations. \\ - The MINPA solar wind data are compared with Earth-based OMNI data. \\ - The blocking effect due to the lander capsule is analyzed quantitatively.
}

Citation: Zhang, A. B., Kong, L. G., Li, W. Y., Li, L., Tang, B. B., Rong, Z. J., Wei, Y., Ma, J. J., Zhang, Y. T., ... and Wang, C. (2022). Tianwen-1 MINPA observations in the solar wind. Earth Planet. Phys., 6(1), 1-9. http://doi.org/10.26464/epp2022014

\begin{abstract}
The Mars Ion and Neutral Particle Analyzer (MINPA) is one of the three scientific instruments onboard the Tianwen-1 orbiter to investigate the Martian space environment. During Tianwen-1's transfer orbit to Mars, the MINPA was switched on to measure the solar wind ions. Here, we present the first results of the MINPA observations in the solar wind. During cruise, nearly half of the MINPA ion fieldof-view (FOV) was blocked by the lander capsule; thus only the solar-wind ions with azimuthal speeds pointing towards the unblocked FOV sectors could be detected. We perform a detailed comparison of the MINPA's solar wind observations with data from Earth-based missions when MINPA reached its count-rate peak, finding a general consistency of the ion moments between them. The blocking effect due to the lander is evaluated quantitatively under varying solar-wind velocity conditions. Despite the blocking effect, the MINPA's solar wind measurements during the transfer orbit suggest a good performance.
\end{abstract}

Keywords: MINPA; Tianwen-1; solar wind; capsule blocking effect

\section{Introduction}

Tianwen-1 is a planetary spacecraft mission to Mars led by the China National Space Administration (CNSA). Its goals are to investigate the planet's surface geology and internal structure, to search for evidence of present and past Martian water, and to characterize the space environment and the atmosphere of Mars (Wei Y et al., 2018; Wan WX et al., 2020a, b; Zou YL et al., 2021).

Correspondence to: A. B. Zhang, zhab@nssc.ac.cn

L. G. Kong, klg@nssc.ac.cn

Received 30 NOV 2021; Accepted 06 DEC 2021.

Accepted article online 09 DEC 2021.

(C) 2022 by Earth and Planetary Physics.
Tianwen- 1 consists of five equipment components: an orbiter, a lander, a deployable camera, a drop camera, and the Zhurong rover. The spacecraft has a total mass of nearly five tons and carries thirteen scientific instruments. Tianwen-1 was successfully launched from the Wenchang Spacecraft Launch Site on 23 July 2020 and entered a Mars orbit on 10 February 2021. The capsule containing a lander and the Zhurong rover separated from the orbiter and entered the Martian atmosphere on 15 May 2021. The lander, which carried the rover, touched down safely in the Utopia Planitia region on Mars. The orbiter has served as a telecommunications relay for the Zhurong rover while continuing to conduct its own orbital observations of Mars. Its target orbit for scientific operations is $265 \mathrm{~km} \times 12,000 \mathrm{~km}$ with an inclination angle 
of $93^{\circ}$.

To explore the Martian space environment, the orbiter is equipped with three scientific instruments, including the Mars lon and Neutral Particle Analyzer (MINPA, Kong LG et al., 2020), the Mars orbiter Magnetometer (MoMAG, Liu K et al., 2020), and the Mars Energetic Particle Analyzer (MEPA, Tang SW et al., 2020). The MINPA was designed and implemented to detect ions and energetic neutral atoms (ENAs) in the Mars space environment. During Tianwen-1's transfer orbit to Mars, MINPA was switched on from 31 October 2020 to 26 January 2021 to measure solar wind ions. Solar wind is the continuous supersonic plasma outflow from the Sun filling the interplanetary space. Generally, the solar wind can be classified into two types according to its speed, fast solar wind and slow solar wind. The fast solar wind is usually connected to the coronal holes on the Sun, while the slow solar wind may emanate from several possible regions, e.g., the edges of active regions, the boundary of a coronal hole, and the quiet regions ( $\mathrm{He}$ JS et al., 2010; Feng XS et al., 2010; Liu YCM et al., 2014; Abbo et al., 2016). In the highly inhomogeneous solar atmosphere, the source regions of the slow and fast streams may be adjacent to one another and at the same latitude. Since the solar wind flows mainly radially outward beyond the Aflvén zone when the flow speed surpasses the local Alfvén speed, it is anticipated that, due to the Sun's rotation, the two streams from adjacent source regions will interact with each other.

When a fast stream behind catches up with and overtakes a slower stream, a compressive interaction region is produced, forming a spiral pattern (as projected onto the solar equatorial plane); such regions are thus known as stream interaction regions (SIRs) (Forsyth and Marsch, 1999; Gosling and Pizzo, 1999; Richardson et al., 2018). If the source regions are long-lived, lasting for over one solar rotation period, the SIR spiral pattern can co-rotate with the Sun, leading to the formation of a co-rotating interaction region (CIR) (Burlaga, 1995). Physically speaking, SIRs are prominent large-scale structures in the solar wind that are a consequence of spatial variability in the coronal expansion and solar rotation. From in-situ data, SIRs can be identified as a density enhancement, a temperature rise, and a flow speed increase. During portions of the transit, MINPA was switched on to measure the solar wind ions, and several SIRs were encountered during those times. Here, we present results of MINPA observations in the solar wind during the transit, and show a detailed comparison between the solar wind data obtained from MINPA and the Earth-based instruments, during an SIR event when MINPA reaches its highest count rate. The blocking effect on the MINPA due to the lander capsule during the transit is also evaluated.

\section{MINPA Observations}

The MINPA instrument integrates ion and ENA measurements into one sensor head to share one electronics box. MINPA utilizes a standard toroidal top-hat electrostatic analyzer (ESA) followed by a time-of-flight (TOF) mass spectrometer to measure ions with energies from $2.8 \mathrm{eV}$ up to $25.9 \mathrm{keV}$ and ENAs from $50 \mathrm{eV}$ to $3 \mathrm{keV}$. For the ENA measurement, highly polished silicon single crystal substrates with an $\mathrm{Al}_{2} \mathrm{O}_{3}$ film coating are adopted (Scheer et al., $2007,2009)$ to ionize neutral particles before they enter the ESA and TOF. MINPA provides a $360^{\circ} \times 90^{\circ}$ field of view (FOV) with $22.5^{\circ} \times 5.4^{\circ}$ angular resolution for the ion measurement and a $360^{\circ} \times 9.6^{\circ} \mathrm{FOV}$ with $22.5^{\circ} \times 9.7^{\circ}$ angular resolution for the ENA measurement. The TOF mass spectrometer can resolve $\mathrm{H}^{+}, \mathrm{He}^{2+}$, $\mathrm{He}^{+}, \mathrm{O}^{+}, \mathrm{O}^{2+}$, and $\mathrm{CO}^{2+}$ ions and $\mathrm{H}$ and $\mathrm{O}(\geqslant 16$ amu group) ENAs. For the solar wind observations during transit to Mars, the ion energy range was adjusted to be from $45 \mathrm{eV}$ up to $24.2 \mathrm{keV}$, with 64 logarithmic energy steps and a time resolution of 4.1 seconds. Due to the ramp-downs of high voltages during the spacecraft maneuvers, the ion measurement unit of the MINPA was operated intermittently; no mass resolution data were collected during these spacecraft activities.

The color-coded background in Figure 1a shows the ambient solar wind radial velocity $V_{r}$ at 15:00 UTC on 23 November 2020 from a heliospheric magnetohydrodynamics (MHD) model. This model was first proposed by Florinski et al. (2013), and more details of this model and the simulation setups can be found in Guo XC and Florinski (2014, 2016), Guo XC et al. (2019), Wang YX et al. (2020), and this report's Supplementary Material. All physical quantities are presented in the Heliographic Inertial (HGl) coordinate system, which is Sun-centered and inertially fixed with respect to an $X$ axis along the intersection line between the ecliptic plane and solar equatorial plane. The $Z$ axis is along the solar rotation axis (positive for northward) and the $Y$ axis completes the right-handed set. In this study, we adopt the corresponding spherical coordinates $(r, \theta, \phi)$.

In Figure 1a, the blue and yellow regions denote slow and fast solar wind, respectively. One can unambiguously identify four SIRs at the transition between the blue region ahead and the yellow region behind. The red curve represents Tianwen-1's Earth-Mars transfer orbit; the blue curves denote the locations of the intermittent MINPA measurements. The locations of Earth, Tianwen-1 (blue circle), and Mars at 15:00 UTC on 23 November 2020 are displayed in Figure 1a. At that time, Earth and Tianwen-1, with $15.7^{\circ}$ azimuthal separation, were located in the same SIR.

The spacecraft axis referring to Figure $1 \mathrm{~b}$ points towards the Sun in the transfer orbit. Figures $1 \mathrm{~b}$ and $1 \mathrm{c}$ show the MINPA and part of the spacecraft viewed from the north and from the Sun at 15:00 UTC on 23 November 2020, respectively. The green and blue shadows denote the unblocked and blocked FOV sectors of the MINPA ion unit, respectively. Sixteen elevation FOV, each of them $5.62^{\circ}$ wide, span the $90^{\circ}$ pole-to-equator range. Sixteen azimuthal FOV sectors, each sector $22.5^{\circ}$ wide, are implemented by sixteen anode pairs, thus covering the complete $360^{\circ}$ azimuthal angle (for more details refer to Kong LG et al. (2020)). As shown in Figure 1b, MINPA is mounted on the $-\boldsymbol{e}_{\varnothing}$ side of the lander capsule. Nearly half of its FOVs $\left(180^{\circ}-360^{\circ}\right.$ azimuthal angle) are thus blocked by the capsule while in transit; solar wind ions directed towards those FOV sectors thus could not be detected by MINPA. In the following, we present the results of the MINPA observations in the solar wind during its transit to Mars.

Figure 2 shows the results of all the MINPA's solar wind observations from 31 October 2020 to 25 January 2021. The omnidirectional differential energy flux shown in Figure $2 a$ has a characteristic feature of cold solar wind ions with total kinetic energies from 

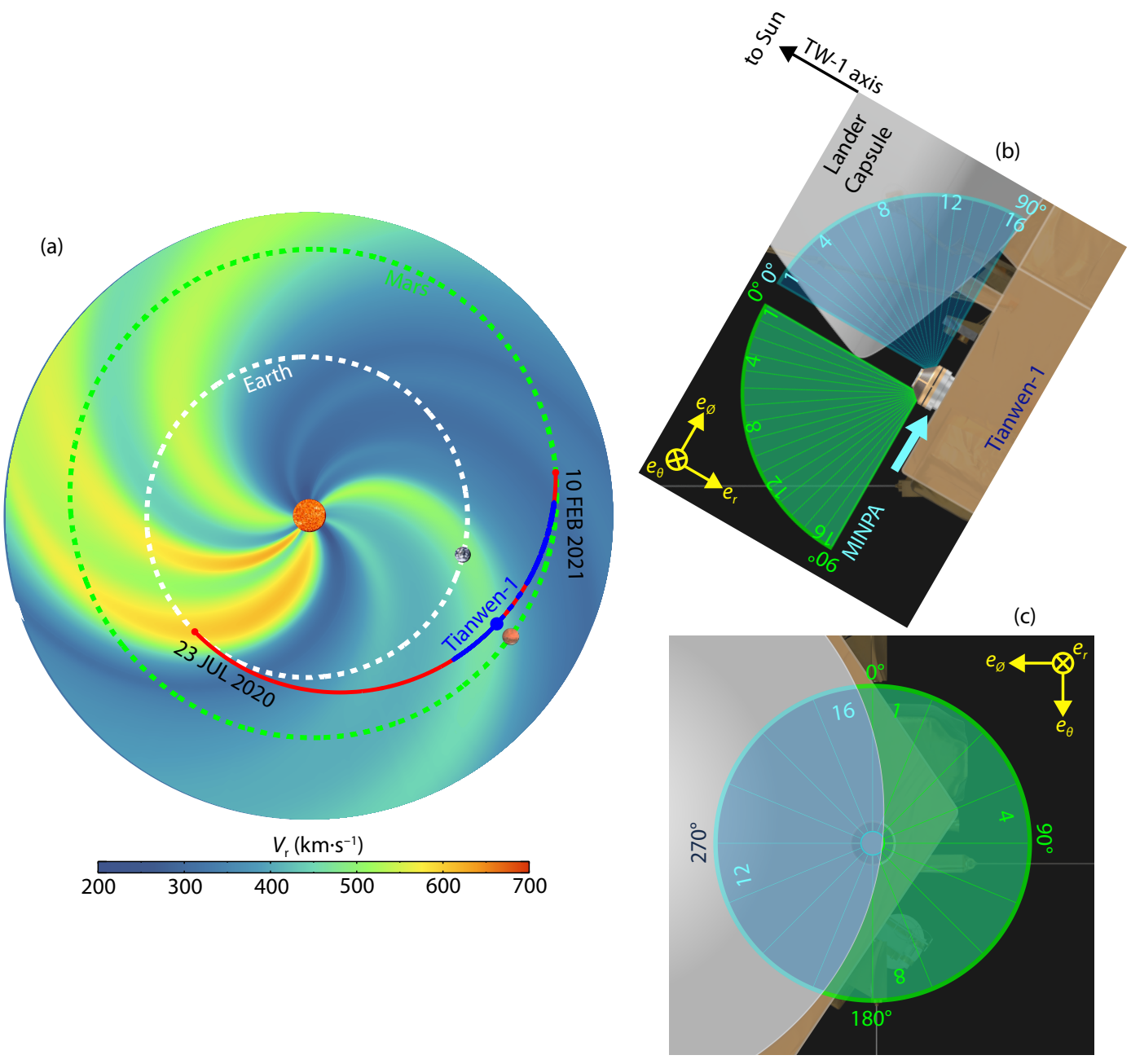

Figure 1. Tianwen-1 transfer orbit from Earth to Mars and MINPA field-of-view (FOV) in the solar wind. (a) Illustration of the solar wind in Heliographic Inertial ( $\mathrm{HGI}$ ) coordinates. Background colors show the solar wind's radial velocity $V_{\mathrm{r}}$ on the solar equatorial plane at 15:00 UTC on 23 November 2020; and the locations of Earth, Tianwen-1 (blue circle), and Mars at this time are also marked. The red curve denotes Tianwen-1's transfer orbit, while the blue curves represent the locations where the MINPA operated in the solar wind mode. (b) Top view (from north to south) and (c) view from the Sun towards Tianwen-1. The green and blue shadows denote the unblocked and blocked FOV sectors of the MINPA ion unit, respectively. Nearly half of the MINPA's FOV sectors are blocked by the lander capsule.

$\sim 500 \mathrm{eV}$ to $\sim 2 \mathrm{keV}$ in the spacecraft frame. To compare with realtime solar wind data from other missions, we plot the OMNI solar wind bulk energies in Figure 2a, while the detailed OMNI parameters are presented in Figures $2 \mathrm{e}-2 \mathrm{~h}$. The OMNI data set is primarily a 1963-to-current compilation of near-Earth solar wind magnetic field and plasma parameter data from several spacecraft in geocentric orbits, including ISEE3, Wind, and ACE (King and Papitashvili, 2005). Here, we use the 5-min high-resolution OMNI data. The unambiguous SIRs are marked in Figure 2f. An interplanetary coronal mass ejection (ICME) hit Earth on 10 December 2020, and a recent study by Mittelholz et al. (2021) analyzed its impact on the magnetic field at the Mars surface using the InSight data. Unfortunately, MINPA was turned off during this time interval and missed this ICME event. As shown in Figure $2 f$, the overall OMNI solar wind speed varies from $250 \mathrm{~km} \cdot \mathrm{s}^{-1}$ to $670 \mathrm{~km} \cdot \mathrm{s}^{-1}$; and the typical ion energies observed by MINPA are consistent with the varying $\mathrm{OMNI}$ ion bulk energies, as shown in Figure 2a.

As the direction of the MINPA $0^{\circ}$ polar angle points towards the
Sun (see Figure $1 \mathrm{~b}$ ), the radial directed solar wind ions are predominantly detected by the MINPA FOVs within the $30^{\circ}$ polar angle, as shown in Figure 2b. MINPA has nearly zero count in the FOV sectors blocked by the lander capsule, with azimuthal angles from $180^{\circ}$ to $360^{\circ}$ (Figure 2c). The black vertical line in Figure 2 indicates the time interval when MINPA reached its highest count rate during the transfer orbit. For this case, we estimate the ion plasma moments from the integral of the measured phase-space densities and present a detailed comparison between MINPA and OMNI data in the following.

Figure 3 presents the detailed observations of MINPA with its highest count rate from 16:00 UTC on 21 November to 10:00 UTC on 22 November 2020, when an SIR swept past Earth and Tianwen-1. As shown by the OMNI data, this SIR event is characterized by a rapid solar wind speed increase (Figure 3e), a small local density peak (Figure 3d), an enhancement of the proton temperature (Figure $3 \mathrm{i}$ ), and a deflection of the solar wind in the transverse direction (Figures $3 \mathrm{f}$ and $3 \mathrm{~g}$ ). MINPA detected solar wind 


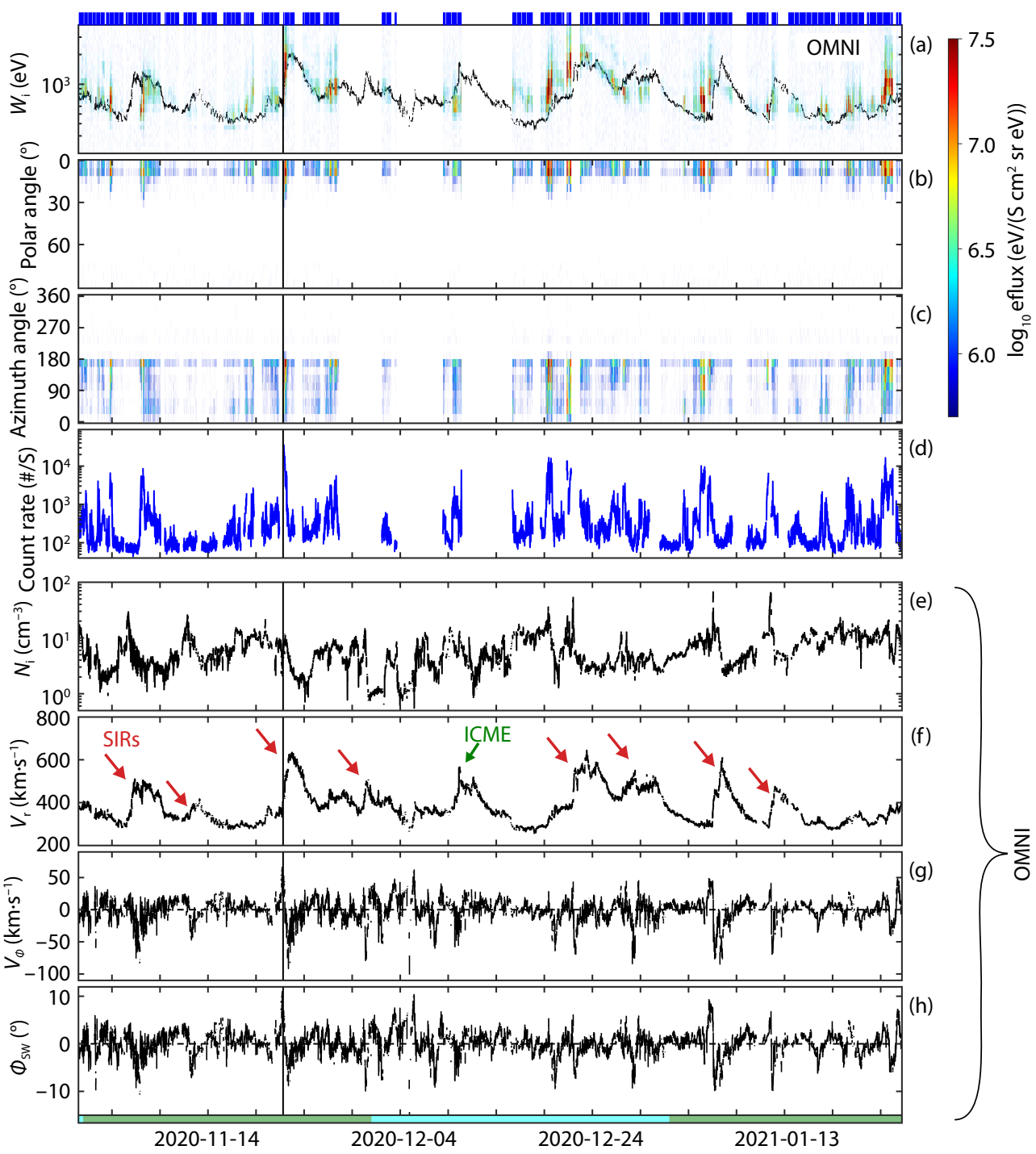

Figure 2. MINPA solar wind observations from 31 October 2020 to 25 January 2021 during the transfer orbit and OMNI solar wind data from Earth-based missions. (a) MINPA ion omnidirectional differential energy flux and OMNI solar wind bulk energy (black curve). MINPA ion energy flux spectrogram along instrumental (b) polar and (c) azimuth angles. (d) MINPA ion count rate. OMNI solar wind data of (e) ion number density $N_{\mathrm{i}}$ (f) radial velocity $V_{\mathrm{r}}$ (g) azimuthal velocity $V_{\Phi}$. (h) Azimuthal transverse angle $\Phi_{\mathrm{SW}}$ defined by arctan $\left(V_{\Phi} / V_{\mathrm{r}}\right)$. The blue bars on top of panel (a) denote the MINPA operating time intervals. The green and cyan bars at the bottom of panel (h) represent different months (October, November, and December of 2020 and January of 2021). The black vertical line in (a)-(h) highlights the interval in the whole transfer orbit when MINPA reached its count-rate peak.

ions entering the high elevation in FOV sectors 1-2, in the unblocked half of azimuthal sectors (Figure 3c). As shown in Figures $3 a-3 b$, the intensity of the solar wind energy flux decreases gradually with time, and the polar angle spread becomes narrower. The azimuthal angles with the energy flux peaks vary from $\sim 90^{\circ}$ to $\sim 180^{\circ}$ (see Figure $3 \mathrm{c}$ ). To further understand those observations, we show the phase-space densities of $1069 \mathrm{eV}$ ions for two data points (at the times indicated by the two vertical lines in Figures 3a-3i). For the first point, a local maximum of phasespace density shown in Figures $3 \mathrm{j}$ is observed by a FOV at $\sim 11^{\circ}$ polar angle and $\sim 110^{\circ}$ azimuth angle, suggesting that MINPA has a nearly complete coverage of solar-wind proton distributions. This can happen only when the solar wind has a positive azimuth- al speed $V_{\varnothing}$ and a negative $V_{\theta}$, which are consistent with the solar wind velocity from $\mathrm{OMNI}$ at the time of Figure $3 \mathrm{j}$ (see Figures $3 e-3 h$ ). From then on, $v_{\varnothing}$ turns from positive to negative, and the solar-wind ions are directed from the unblocked to blocked FOV sectors. As an example, the observed phase-space density peak in Figure $3 \mathrm{k}$ locates at the $180^{\circ}$ azimuth-angle boundary of the unblocked FOVs. As the solar wind continues to turn towards $-V_{\varnothing}$ directions, most of the ions are blocked by the lander capsule, which agrees well with the rapid decrease of the detected solar wind fluxes (Figures 3a-3c).

The nearly complete measurement of solar wind ions in part of the SIR event shown in Figure 3 allows us to perform a comprehensive comparison of the solar wind data between MINPA and 


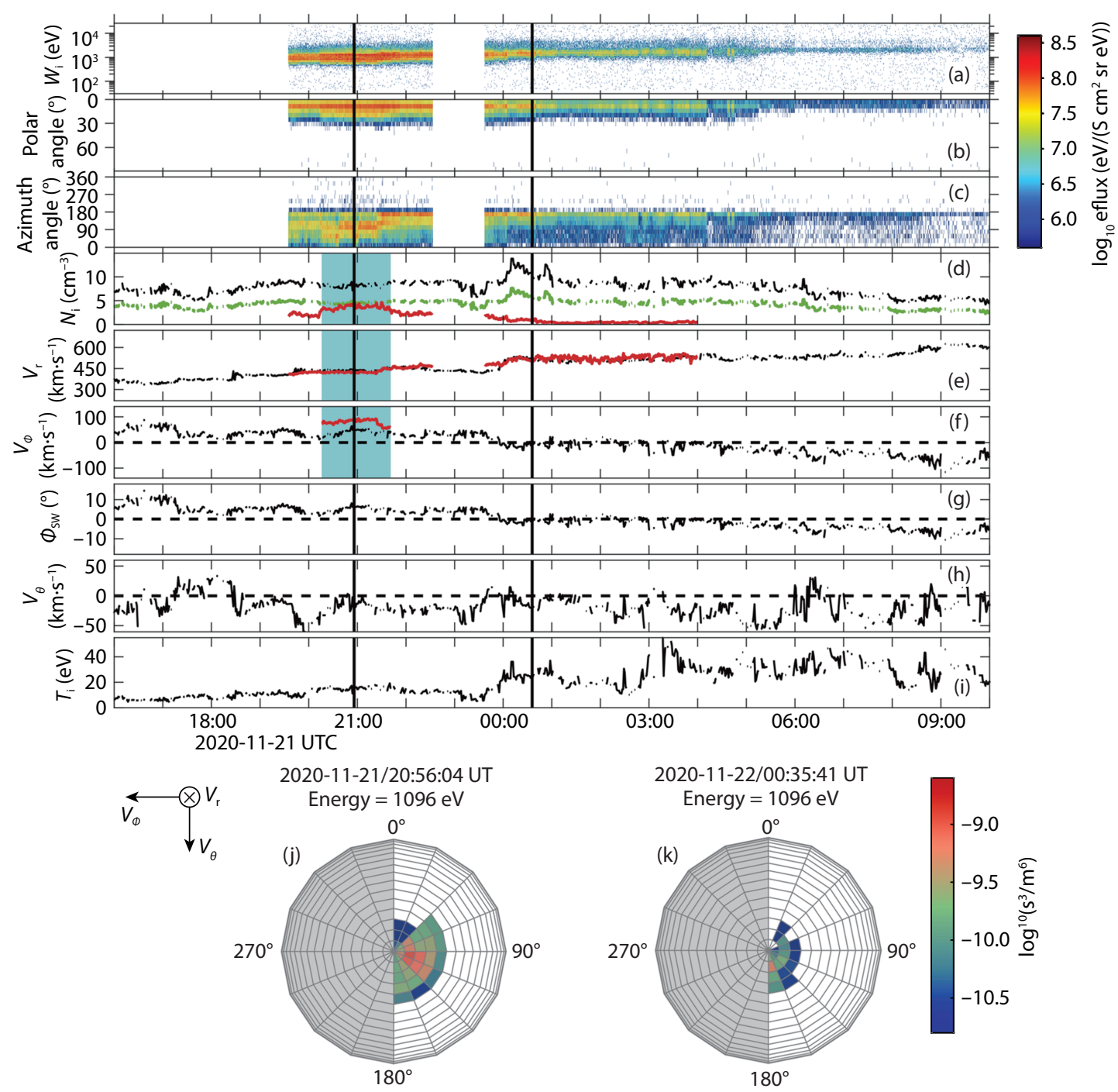

Figure 3. MINPA solar-wind observations during an SIR event. Panels show MINPA measured omnidirectional energy flux as a function of (a) energy, (b) polar angle, and (c) azimuth angle. Solar wind parameters of (d) $N_{\mathrm{i},}$ (e) $V_{\mathrm{r}}$, (f) $V_{\Phi}$, (g) $\Phi_{\mathrm{SW}}$, (h) $V_{\theta}$, and (i) $T_{\mathrm{i}}$. The black curves in (d) (i) show the corresponding OMNI data; the red curves in (d)-(f) are MINPA ion moments. The green curve in panel (d) shows the inferred number density at Tianwen-1. We show $V_{\Phi}$ only with MINPA $N_{\mathrm{i}}$ larger than $3 \mathrm{~cm}^{-3}$, indicated by the cyan bar in (d) - (f). Panels (j) and (k) present the phase-space density skymaps of $1096 \mathrm{eV}$ ions viewed from the Sun at times marked by the two vertical black lines in (a)-(i).

OMNI. We compute the ion plasma moments from the integral of the phase-space densities measured by the MINPA; the number density and the radial and azimuthal speeds are presented by the red curves in Figures 3d-3f, respectively. The MINPA radial speed has an increasing tendency similar to that from the OMNI data. Even though a significant part of the ion distribution functions are blocked by the lander capsule, $V_{r}$ from MINPA is still relatively reliable because of the low-temperature nature of solar-wind protons. The time delay between MINPA and OMNI $V_{r}$ is close to zero from the least squares fitting method. This time delay is much shorter than the usual solar wind propagation time ( 30 hours) from the Earth orbit (1 AU) to Tianwen-1 ( 1.34 AU), which is attributed to the spiral shape of the SIR, which enable the simultaneous observation at Earth and by MINPA (see Figure 1a). This negligible time delay suggests that we can compare the OMNI data with the solar wind data recorded at Tianwen-1.
Because the solar wind number density roughly satisfies an inverse square falloff with increasing heliocentric distance (Liu D et al., 2021), the OMNI number density should be decreased by a factor of 0.55 when reaching Tianwen-1. As shown in Figure 3d, the MINPA number density (red curves) is clearly different from the inferred OMNI number density (green curve). When MINPA has a nearly complete coverage of the solar wind distribution (the cyan-colored region in Figure 3), the number density from MINPA is in good agreement with the inferred value. In the second interval of MINPA measurement in the time of Figure 3, the MINPA number density is much smaller than the inferred value. The coexistence of the density variation of MINPA and the deflection of the solar wind transverse speed provides a good opportunity to assess the blocking effect by the lander capsule quantitatively.

\section{Quantification of Blocking by the Lander Capsule}

Figure 4 presents the unblocking ratios of the observed number 
density to the background one as a function of the solar-wind azimuthal speed $V_{\Phi}$. Here, we assume that the $-\boldsymbol{e}_{\Phi}$ side of MINPA FOV sectors are fully blocked by the lander capsule. The blue and black dashed curves show the blocking ratios of two radial moving $\left(400 \mathrm{~km} \cdot \mathrm{s}^{-1} V_{\mathrm{r}}\right.$ ) Maxwellian distribution functions with ion temperatures of $15 \mathrm{eV}$ and $25 \mathrm{eV}$, respectively. A small deflection (e.g., $5^{\circ}$ ) of the transverse angle $\Phi_{\mathrm{SW}}$ yields a significant variance in the blocking ratios. The black crosses show the number density ratios of the MINPA results to the inferred OMNI ones and their variance along the OMNI $V_{\Phi}$. The cyan bar in Figures $3 d-3 f$ indicates the interval with MINPA $N_{i}$ larger than $3 \mathrm{~cm}^{-3}$, where major parts of the ion distributions are detected, and the red circles in Figure 4 present the blocking ratios of this interval using the MINPA $V_{\Phi}$. In general, the MINPA blocking ratios follow the trend of those of the Maxwellian distributions, though most of them are lower than the predicted ones. This difference is likely to reflect the reality that the blocking of the FOV sectors in reality is slightly larger than one half of the MINPA's $2 \pi$ FOV (Figure 1c). Besides, the MINPA's transverse speeds (the cyan interval in Figures $3 d-3 f$ or the red circles in Figure 4) are over-estimated due to the missing measurement of the $-V_{\Phi}$ part of the phase-space densities. When an SIR sweeps past Tianwen-1, a positive azimuthal speed is beneficial for the solar wind measurement by MINPA. Because of the largest positive transverse angle $\Phi_{\text {SW }}$ (see Figure $2 \mathrm{~h}$ ) occurred in the time interval of Figure 3, MINPA received its highest count rate. From Figure 2, one can also find other intervals with relatively high count rate when the solar wind has positive $V_{\Phi}$. Despite the blocking effect, MINPA had a relatively good performance of the solar-

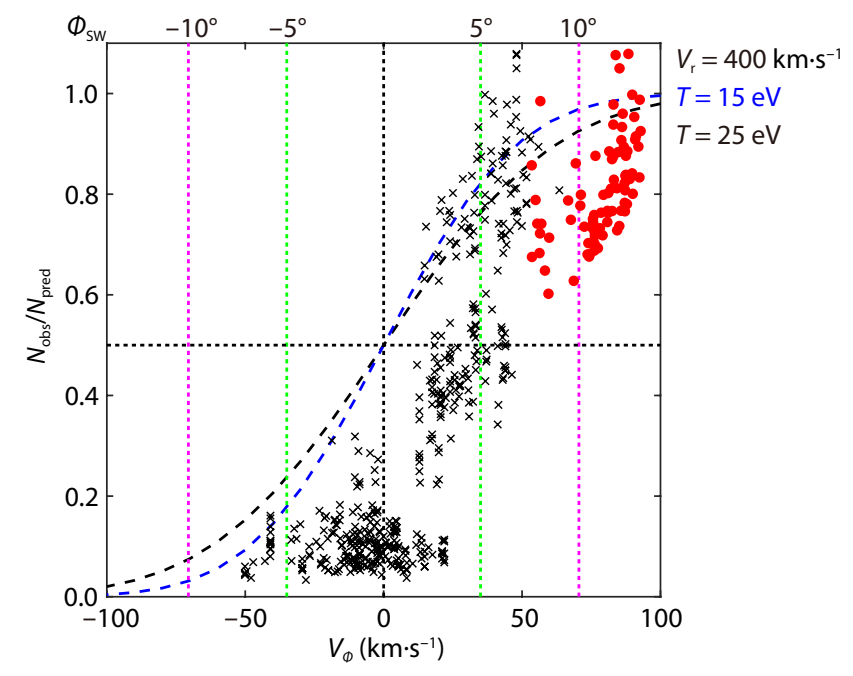

Figure 4. Dependence of the capsule blocking effect on the transverse component of the solar wind speed $V_{\Phi}$. We assume that the $-\boldsymbol{e}_{\Phi}$ side of MINPA FOV sectors are fully blocked by the lander capsule. The black crosses represent the ratios of the MINPA number density to the inferred OMNI number density, as a function of the OMNI $V_{\Phi}$. The red circles denote the ratios where the measured MINPA $N_{\mathrm{i}}$ is larger than $3 \mathrm{~cm}^{-3}$ and use the MINPA $V_{\Phi}$. The blue and black dashed curves represent the blocking ratios of two Maxwellian distribution functions with temperatures of $15 \mathrm{eV}$ and $25 \mathrm{eV}$, respectively. wind measurement during the Earth-Mars transfer orbit.

\section{Summary}

Tianwen-1, China's first spacecraft mission to Mars, was successfully launched on 23 July 2020 and entered a Mars orbit on 10 February 2021. During portions of the transit, MINPA was switched on to measure the solar wind ions. Several SIRs were encountered during those times. The characteristic energies of the ions observed by MINPA are similar to those from Earth-based OMNI data set. The MINPA's $0^{\circ}$ polar angle was oriented towards the Sun; solar wind ions flowing radially were thus the ones preferentially detected by unblocked MINPA sectors with polar angles smaller than $30^{\circ}$. The azimuthal FOVs from $\sim 180^{\circ}$ to $360^{\circ}$ were blocked by the lander capsule. As a result, MINPA recorded nearly zero count in those FOV sectors. We performed a detailed comparison between the solar wind data obtained from MINPA and OMNI, during an SIR event when MINPA's count rate was highest during the transfer phase of Tianwen-1's journey to Mars. In the intervals characterized by positive solar-wind transverse speed, most solar wind ions were able to enter into the MINPA's unblocked FOV sectors; data from such times confirm that the ion number density and speed integrated from MINPA-measured phase-space densities are consistent with the ion moments inferred from OMNI data. When solar wind azimuthal speed turned to be negative, the MINPA-measured solar wind counts dropped dramatically. We used these data to quantify, under several azimuthal speed conditions, the effects of the lander capsule on blocking MINPA. Despite the blocking, MINPA performed relatively well in measuring the solar wind during Tianwen-1's trip to Mars.

\section{Acknowledgements}

We acknowledge China National Space Administration (CNSA), Ground Research and Application System (GRAS), Beijing Aerospace Command and Control Center (BACC), and China Academy of Space Technology (CAST). We thank all the MINPA team members for their dedicated work. We appreciate Dr. Xueshang Feng, Dr. Fang Shen, Dr. Hui Li, Dr. Yong Liu, and Limin Wang from NSSC and Xingyu Zhu, Chuanpeng Hou, Rong Lin from Peking University for the scientific discussion and Linrong Wu for the cartoon visualization. This work was supported by the Key Research Program of the Chinese Academy of Sciences (Grant NO. ZDBS-SSW-TLC00103), Strategic Priority Research Program (B) of the Chinese Academy of Sciences (XDB41000000), NNSFC Grant No. 41974170 and 41974196, Specialized Research Fund for State Key Laboratories of China, and Pandeng Program of National Space Science Center, Chinese Academy of Sciences. BBT (2019153), YTZ (2017186), WYL (2018177), and LHX (2021144) were supported by the Youth Innovation Promotion Association, and WYLI was also funded by the Young Elite Scientists Sponsorship Program by CAST. The OMNI data were generated by J. $\mathrm{H}$. King and N. Papitashivilli and provided via http://cdaweb.gsfc. nasa.gov/.

\section{References}

Abbo, L., Ofman, L., Antiochos, S. K., Hansteen, V. H., Harra, L., Ko, Y. K., Lapenta, G., Li, B., Riley, P., ... Wang, Y. M. (2016). Slow solar wind: Observations and 
modeling. Space Sci. Rev., 201(1), 55-108. https://doi.org/10.1007/s11214016-0264-1

Arge, C. N., Odstrcil, D., Pizzo, V. J., and Mayer, L. R. (2003). Improved method for specifying solar wind speed near the Sun. AIP Conf. Proc., 679, 190-193. https://doi.org/10.1063/1.1618574

Burlaga, L. F. (1995). Interplanetary Magnetohydrodynamics. Oxford: Oxford University Press.

Feng, X. S., Yang, L. P., Xiang, C. Q., Wu, S. T., Zhou, Y. F., and Zhong, D. K. (2010). Three-dimensional solar wind modeling from the Sun to Earth by a SIPCESE MHD model with a six-component grid. Astrophys. J., 723(1), 300-319. https://doi.org/10.1088/0004-637x/723/1/300

Florinski, V., Guo, X., Balsara, D. S., and Meyer, C. (2013). Magnetohydrodynamic modeling of solar system processes on geodesic grids. Astrophys. J. Suppl. Ser., 205(2), 19. https://doi.org/10.1088/0067-0049/205/2/19

Forsyth, R. J., and Marsch, E. (1999). Solar origin and interplanetary evolution of stream interfaces. Space Sci. Rev., 89(1), 7-20. https://doi.org/10.1023/ A:1005235626013

Gosling, J. T., and Pizzo, V. J. (1999). Formation and evolution of corotating interaction regions and their three dimensional structure. In A. Balogh, et al. (Eds.), Corotating Interaction Regions (pp. 21-52). Dordrecht: Springer. https://doi.org/10.1007/978-94-017-1179-1_3

Guo, X., and V. Florinski. (2014). Corotating interaction regions and the 27 day variation of galactic cosmic rays intensity at $1 \mathrm{AU}$ during the cycle 23/24 solar minimum. J. Geophys. Res. :Space Phys., 119(4), 2411-2429. https://doi.org/10.1002/2013ja019546

Guo, X., and Florinski, V. (2016). Galactic cosmic-ray intensity modulation by Corotating interaction region stream interfaces AT 1 Au. Astrophys. J., 826(1), 65. https://doi.org/10.3847/0004-637x/826/1/65

Guo, X. C., Florinski, V., and Wang, C. (2019). A global MHD simulation of outer heliosphere including anomalous cosmic-rays. Astrophys. J., 879(2), 87. https://doi.org/10.3847/1538-4357/ab262b

He, J. S., Marsch, E., Tu, C. Y., Guo, L. J., and Tian, H. (2010). Intermittent outflows at the edge of an active region-a possible source of the solar wind. Astron. Astrophys., 516, A14. https://doi.org/10.1051/0004-6361/200913712

King, J. H., and Papitashvili, N. E. (2005). Solar wind spatial scales in and comparisons of hourly Wind and ACE plasma and magnetic field data. J. Geophys. Res. :Space Phys., 110(A2), A02104. https://doi.org/10.1029/ 2004JA010649

Kong, L. G., Zhang, A. B., Tian, Z., Zheng, X. Z., Wang, W. J., Liu, B., Wurz, P., Piazza, D., Etter, A., ... Sun, Y. Q. (2020). Mars lon and Neutral Particle Analyzer (MINPA) for Chinese mars exploration mission (Tianwen-1): design and ground calibration. Earth Planet. Phys., 4(4), 333-344. https://doi.org/10.26464/epp2020053

Liu, D., Rong, Z. J., Gao, J. W., He, J. S., Klinger, L., Dunlop, M. W., Yan, L. M., Fan, K., and Wei, Y. (2021). Statistical properties of solar wind upstream of Mars: MAVEN observations. Astrophys. J., 911(2), 113. https://doi.org/10.3847/ 1538-4357/abed50

Liu, K., Hao, X. J., Li, Y. R., Zhang, T. L., Pan, Z. H., Chen, M. M., Hu, X. W., Li, X., Shen, C. L., and Wang, Y. M. (2020). Mars orbiter magnetometer of China's first mars mission Tianwen-1. Earth Planet. Phys., 4(4), 384-389. https://doi.org/10.26464/epp2020058

Liu, Y. C. M., Huang, J., Wang, C., Klecker, B., Galvin, A. B., Simunac, K. D. C., Popecki, M. A., Kistler, L., Farrugia, C., ... Jian, L. (2014). A statistical analysis of heliospheric plasma sheets, heliospheric current sheets, and sector boundaries observed in situ by STEREO. J. Geophys. Res. :Space Phys., 119(11), 8721-8732. https://doi.org/10.1002/2014JA019956

Mittelholz, A., Johnson, C. L., Fillingim, M., Joy, S. P., Espley, J., Halekas, J., Smrekar, S., and Banerdt, W. B. (2021). Space weather observations with InSight. Geophys. Res. Lett., 48(22), e2021GL095432. https://doi.org/10.1029/2021GL095432

Richardson, I. G. (2018). Solar wind stream interaction regions throughout the heliosphere. Living Rev. Sol. Phys., 15, 1-95. https://doi.org/10.1007/s41116017-0011-z

Scheer, J. A., Wahlström, P., and Wurz, P. (2007). Scattering of light molecules from $\mathrm{Al}_{2} \mathrm{O}_{3}$ surfaces. Nucl. Instrum. Methods Phys. Res. Sect. B:Beam Interact. Mater. Atoms, 256(1), 76-80. https://doi.org/10.1016/j.nimb.2006.11.093

Scheer, J. A., Wahlström, P., and Wurz, P. (2009). Scattering of light molecules from thin $\mathrm{Al}_{2} \mathrm{O}_{3}$ films. Nucl. Instrum. Methods Phys. Res. Sect. B:Beam Interact. Mater. Atoms, 267(16), 2571-2574. https://doi.org/10.1016/j.nimb. 2009.05.016

Tang, S. W., Wang, Y., Zhao, H. Y., Fang, F., Qian, Y., Zhang, Y. J., Yang, H. B., Li, C. H., Fu, Q., ... Sun, Z. P. (2020). Calibration of Mars Energetic Particle Analyzer (MEPA). Earth Planet. Phys., 4(4), 355-363. https://doi.org/10.26464/ epp2020055

Wan, W. X., Wang, C., Li, C. L., and Wei, Y. (2020a). China's first mission to Mars. Nat. Astron., 4(7), 721-721. https://doi.org/10.1038/s41550-020-1148-6

Wan, W. X., Wang, C., Li, C. L., Wei, Y., and Liu, J. J. (2020b). The payloads of planetary physics research onboard China's First Mars Mission (Tianwen-1). Earth Planet. Phys., 4(4), 331-332. https://doi.org/10.26464/epp2020052

Wei, Y., Yao, Z. H., and Wan, W. X. (2018). China's roadmap for planetary exploration. Nat. Astron., 2(5), 346-348. https://doi.org/10.1038/s41550018-0456-6

Wang, Y. M., and Sheeley, Jr. N. R. (1990). Solar wind speed and coronal fluxtube expansion. Astrophys. J., 355, 726-732. https://doi.org/10.1086/168805

Wang, Y. X., Guo, X. C., Wang, C., Florinski, V., Shen, F., Li, H., and Blanc, M. (2020). MHD modeling of the background solar wind in the inner heliosphere from 0.1 to $5.5 \mathrm{AU}$ : comparison with in situ observations. Space Wea., 18(6), e2019SW002262. https://doi.org/10.1029/2019SW002262

Zou, Y. L., Zhu, Y., Bai, Y. F., Wang, L. G., Jia, Y. Z., Shen, W. H., Fan, Y., Liu, Y., Wang, C., ... Peng, Y. Q. (2021). Scientific objectives and payloads of Tianwen-1, China's first Mars exploration mission. Adv. Space Res., 67(2), 812-823. https://doi.org/10.1016/j.asr.2020.11.005 


\section{Supplementary Materials for "Tianwen-1 MINPA observations in the solar wind"}

\section{Heliospheric MHD Model}

\subsection{Model Description}

The heliospheric MHD model used in this study was first proposed by Florinski et al. (2013), where multiple populations of plasma and neutral particles can be simulated simultaneously. An improvement to the model with a new treatment of the inner boundary conditions at $0.1 \mathrm{AU}$ was implemented for prediction of the large-scale structures from 0.1 to $5.5 \mathrm{AU}$ in the inner heliosphere from 0.1 to $5.5 \mathrm{AU}$ (Wang YX et al., 2020). The model uses synoptic magnetogram maps as input to derive the inner boundary conditions, based on empirical relations such as the WangSheeley-Arge (WSA) relation (Wang YM and Sheeley, 1990; Arge et al., 2003). The performance of the model was validated by multispacecraft data obtained from OMNI, Solar Terrestrial Relations Observatory (STEREO), Ulysses, Juno, and MErcury Surface, Space ENvironment, GEochemistry, and Ranging (MESSENGER) at different latitudes and heliocentric distances, which have collectively demonstrated the capabilities of this model in predicting the solar wind in the inner heliosphere. The model simulates the background solar wind in the heliographic coordinate system (corotating with the Sun), and assumes a static photospheric magnetic field during each Carrington rotation. This model thus simulates the steady background solar wind. It does not include the effects of solar wind transient events such as ICMEs. A more detailed description of the model can be found in Guo et al. (Guo $X$ and Florinski, 2014, 2016; Guo XC et al., 2019) and Wang YX et al. (2020).

\subsection{Simulation Setups}

The model solves the ideal MHD equations in a three-dimensional grid system, which is composed of a two-dimensional geodesic unstructured grid on a sphere and a concentric nonuniform radial grid (Florinski et al., 2013). In this study, the total grid number of the nonuniform radial grid is 1004, while Level 7 geodesic grids with a total of 40962 faces in the 2-D sphere are utilized to simulate the global heliosphere from 0.1 to $5.5 \mathrm{AU}$, covering the orbits of the Earth, Mars, and the Tianwen-1 spacecraft studied in this work. As a result, the equivalent grid resolution is about $1.25^{\circ}$ in both longitude and latitude, corresponding to a time resolution of $\sim 2.1$ hours.

\subsection{Simulation Results}

Figure S1 compares simulation results with solar wind number densities (a) and radial velocities (b) observed at Tianwen-1 (MINPA) from October 31, 2020 to January 25, 2021. Note that the MHD data cover only the time between October 31, 2020 and December 25, 2020. The observed solar wind data at Earth (i.e., from OMNI data, in blue) are also added for reference. The MINPA time resolution is 4.1 seconds, while the OMNI and MHD data have a time resolution of about 1 minute. Here the original simulation data, with a time resolution of $\sim 2.1$ hours generated by the heliospheric model, are interpolated in order to adapt to the OMNI resolution for direct comparison, which does not affect the results and conclusions in this study. The solar wind number density generally decreases with heliocentric distances $(r)$ as $r^{-2}$, while the radial velocity remains nearly constant during the solar wind's radial propagation in the inner heliosphere. As shown in Figure 1a, during the time covered in Figure S1, Tianwen-1 was located at a heliocentric distance of about 1.4 AU with a separation angle of about $14^{\circ}$ in heliographic longitudes relative to the Earth at $1 \mathrm{AU}$.

As mentioned above, Tianwen-1 was located at a heliocentric distance of about 1.4 AU, which means, theoretically, that the mag-

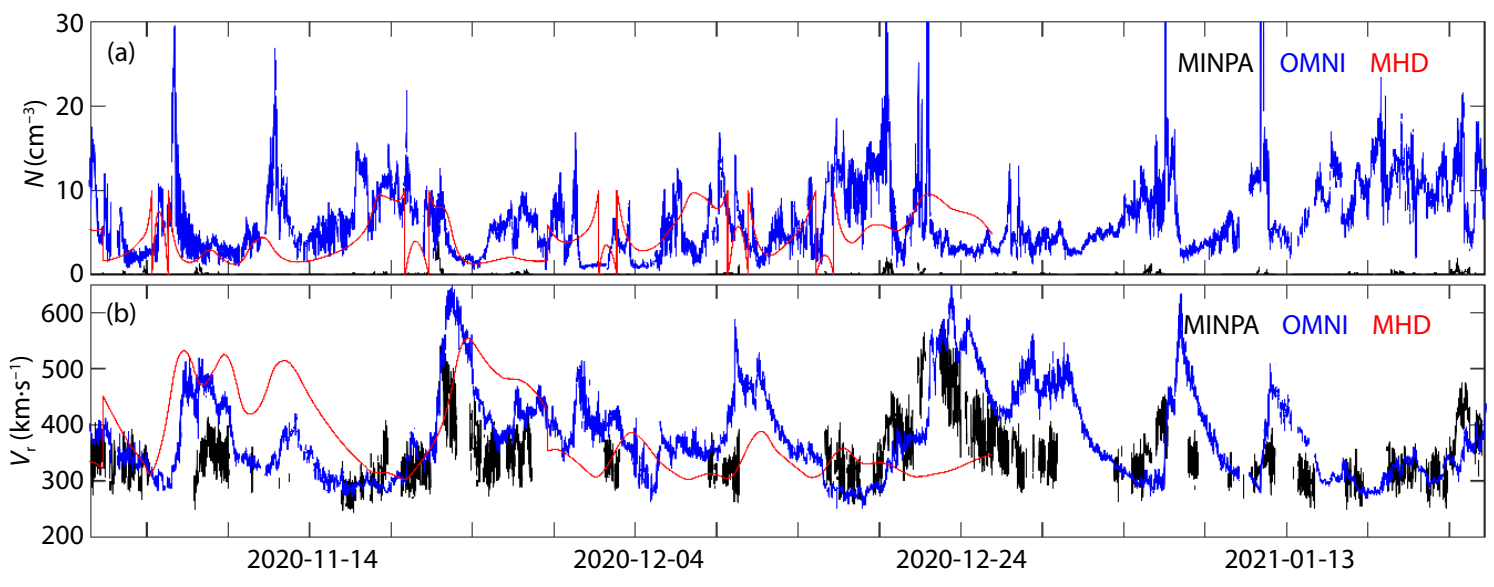

Figure S1. Comparison of the solar wind number densities (a) and radial velocities (b) observed at Tianwen-1 with simulation results (in red). The solar wind parameters at Earth (blue) are obtained from the OMNI database. 
nitude of the number density at Tianwen-1 during this period should be about one half of that at Earth (i.e., from OMNI data). As can be seen in Figure S1a, the number density measured by MINPA is significantly lower than one half the values from OMNI data, which is caused by the shielding effects by the lander capsule. As expected, the magnitudes of the simulated density at Tianwen-1 are in general lower than those observed at Earth, and likely follow the $r^{-2}$ law during certain periods such as December 14, 2020 to December 19, 2020. In addition, the general trend of the simulated density agrees quite well with OMNI data during periods such as between November 14, 2020 and November 24, 2020, although there are also some phase differences during this time.
As can be seen from Figure S1b, the solar wind's radial velocity is comparable at Earth and at Tianwen-1, which is within expectations as mentioned above. The magnitudes and general trends of velocity obtained from the MHD model, from OMNI and from MINPA are consistent during some time periods, such as November 16, 2020 to November 24, 2020. Moreover, the velocity observed at Tianwen-1 seems to follow the OMNI data, with some phase differences during some periods. Nevertheless, large discrepancies can be noted occasionally between the simulations and observations, which could be caused by the limitations of the model such as its grid resolution or the temporal effects, which are not included in the heliospheric model (Wang YX et al., 2020). 